\title{
Research on the Application Value of Hand-painted Art in the New Media Advertising Design
}

\author{
YangJing \\ Jiujiang University, Jiujiang 332005, China
}

Keywords: Hand drawn art, Media advertising, Creative mining, Value.

\begin{abstract}
With the rapid development of modern advanced science and technology, advanced science and technology affects our life style and production mode in various ways, and the rapid development of new media forms the new media era of the digital feature such as Internet and mobile terminal, it continues to impact on the newspapers, periodicals, broadcasting, television and other traditional media. With the development of new technology, the new media has brought great convenience to people, for example, media TV on public transport, mobile phone APP, Internet video and so on bring the full range of influence, which are causing a visual change and changing the social life. By way of image transfer information, the visual communication in new media is the formation of the a new era of reading pictures, changing the aesthetic ability in the image art. Under the background of new media era, the old art has eclipsed, so artists began to pursue high-level information media channels, and designers seek hand-painted art with its unique creativity. Through the innovative mining of hand-painted art, this paper mainly studies the communication value and application significance in the new media advertising design and the purpose is to confirm that the hand drawn art has three aspects of cultural heritage, art and business in the design of new media advertising.
\end{abstract}

\section{Introduction}

With the popularization of computer, digital technology in the field of information has gradually become the core technology of all kinds of media, which brings people a new audio-visual experience. People's daily life is closely related to the Internet, and people get information to become more convenient way [1-3]. With the popularity of the network, the digital graphics can becopied and easy to handle, so that the digital technology is increased in the application of new media advertising design, but also to bring the audience visual aesthetic fatigue, which greatly affect the advertising effectiveness [4,5]. To improve the influence of new media advertising, people can be combined with hand drawn art and digital technology, to add the personalized and novel components of the hand drawn graphics, and then to reduce the visual aesthetic fatigue caused by repeated visual information, which will bring new vitality for the new media advertising and bring new development prospects.

\section{The Characteristics of New Media Advertising}

With the rapid development of Internet technology, making the communication between people and information transfer are more convenient, and the development of digital and multimedia technology has accelerated the transformation of advertising industry, which is the formation of a new kind of media advertising [6-8]. The new kind of media advertising has clear picture, strong hyperopia, content rich, fast and accurate, real-time efficient characteristics, etc., so it can be quickly to seize the market, and will become the development trend of the future market. The characteristics of new media advertising are mainly the following three points [9-11]:

(1) The two-way interaction of audience orientation

As the information transmission platform, advertising generally need to pursue interactive. The effect of media advertising interaction will be high intensity participated by many advertising communication activities, to participate directly in the advertising communication activities are not just the general page view and free advertising click, but also to participate with a sharp point or strong interest. Today, the network is highly developed, advertising has become a two-way 
interactive communication, and new media advertising in the network era makes the theory audience oriented become reality. The new media advertising will be transferred to the role, the author can make and publish different information for different audiences, and the audience can receive information according to their own needs, which has a very clear audience oriented interactive.

(2) The link of information services

Traditional advertising is generally mandatory, and it often force the audience's visual nerve with short ads. However, the new media advertising generally carries out search results based on target users, resulting business product information and services in the new media advertising. People query information in the Internet, they are generally through the specific network port access, and then they can follow the information port to obtain a deeper level of information. So, new media advertising can show the link of information services according to real-time network information transmission link.

(3) The real-time information management

New media advertising and traditional advertising has a subversive distinction, the new media advertising is no longer a static commodity information in the context of the Internet, but is interactive, users can take the initiative to get the brand or product information according to their own needs. So, according to the specific needs of the audience, new media advertising can provide real-time and accurate information to meet the needs of users. The new media advertising not only can carry out the horizontal transmission of all kinds of information, but also can carry out real-time information management.

\section{Hand Drawn Art Forms and its Visual Features}

In the information age, the modern society is put up "reading age ", "information age", "digital era" label because of the multimedia technology rapid development, and modern society has happened great changes, which just shows hand-painted art value [11,12]. Media advertising design is to convey information based on graphic visual elements, and graphics in the social life are kind of visual symbols to convey information, which are more intuitive and easy to understand. With the computer age of science and technology development, the art of hand drawn graphics is a unique artistic charm with its unique form and features.

The development process and its form of modern hand drawn art history. As a kind of transferring information hand-painted graphics, it refers to a method of drawing a graph, which usually refers to the plan diagram for the initial stage of the pattern or the picture design and the effect diagram after the design is finished. Hand drawn art the design of the initial idea and the process of the final completion, including point line, color and other elements. Human society has been through the process of information transmission through the evolution of manual, machine and industrial age. Graphics were the first to appear in the text, and people used graphics to record and convey information in the ancient times [13]. Before the network is not highly developed, the art of hand drawn graphics is always the main way of designing, and when the Internet is slowly filled in the corners of social life, the computer language will be gradually developed. The computer language brings convenient and efficient, but people start to have a strong visual fatigue for the multimedia advertising. At this point, it has a new opportunity for the human hand drawn pattern. Hand drawn image art has been recognized by everyone with its unique personalized, novel and rich human touch, which meets the needs of the new era audience and becomes the darling of the internet era.

Hand drawn art is a unique artistic creation performance, it is more casual with a personalized, interesting and rich human touch, which can be more intuitive to show the author's creative intent. Traditional hand drawn graphics generally refers to a pen in the paper or other media to draw the graphics, hand drawn graphics generally refers to the use of computer software in today. Combined with the drawing board or mouse, people can be freehand graphics as shown in Figure 1. 


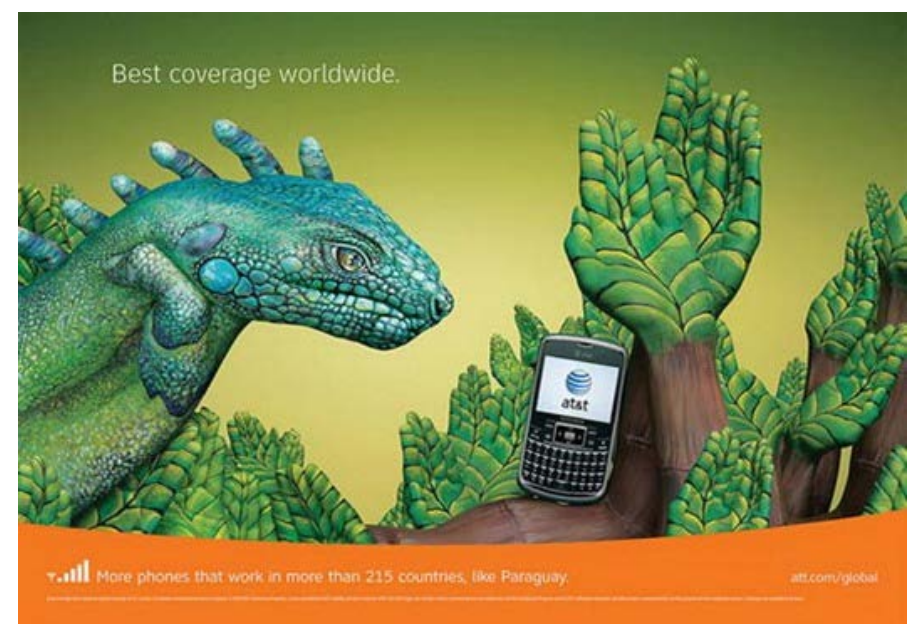

Fig. 1 Aclassic mobile phone creative hand painted advertising

The visual features of modern hand drawn art. As an important organ of human being, the eye is mainly used to obtain information. Through the stimulation of light, the eyes are excited, and the information is processed by the visual system. Through vision, people and animals can get the outside world's size, light, color, movement and other important information. Researchers say that at least $80 \%$ of outside information is acquired by visual. In the society, there are a lot of graphic language, each object has its own image symbol language. Through the graphics language, hand drawn designers convey their ideas to the audience, and graphics language as a bridge or medium is the interpretation of the work meaning and significance. Before the computer, people mainly rely on hand drawn graphics to convey and record information, and in modern hand drawn graphics art visual features is mainly in the form of "shape" and "color", which generally include functional structure and semantic expression.

\section{The Innovation Application and Value Embodiment of Hand Drawn Art in the New Media Advertising Design}

Hand drawn graphics art has rich original flavor and creativity, deep national spirit charm and unique artistic appeal. With the development of the internet technology, making the media advertising design is full of stereotyped "network image", to greatly improve the repeatability. In this context, the audience began to pursue personalized advertising design, and hand drawn art began to be sought by the designer $[14,15]$. From the computer design software back to the traditional hand drawn art, hand drawn art shows its unique cultural heritage, art aesthetic and commercial marketing value in the new media era.

The perspective of cultural heritage. Many years ago, there was a hand drawn art, which is mainly used for communication and information transfer, and communication and information transfer is a very important part of human being. Therefore, the development and inheritance of the hand drawn art is an inevitable process. However, with the development of the network, designers are beginning to like high efficiency and easy to use drawing software as a design tool, and hand drawn art starts to gradually withdraw from the art design circle. In addition, the use of hand-painted art in the new media advertising design can be known that the designer is only simple copying for hand-painted techniques, and they are lack of innovation. Therefore, the use of hand drawn graphic art should pay attention to innovative applications in the design of new media advertising.

Chinese national culture is profound, five thousand years of cultural precipitation is the legacy of our ancestors. In the design of art, we should pay attention to the excavation and the absorption of the national culture, and pursuit or create the traditional culture immensely, and use the essence of the traditional culture in the contemporary design. Today, the competition is fierce, with continuous innovation and development, the modern hand drawn art can be long-term heritage and carry forward when the traditional culture is pushed to a new design stage. The application of hand drawn art in the design of the traditional advertising is widely, new media advertising is the development of the formation of the traditional culture, and hand drawn art is the innovation and development of 
traditional culture, therefore, the application of hand drawn art in the new media advertising has a relatively deep significance in the heritage of national culture.

The perspective of art aesthetic. The value of each piece of art is reflected in its aesthetic degree, and the study of artistic value designs to sell the works of mass satisfaction based on the social aesthetic pursuit. Things with beauty can touch the kind of spiritual moving, and this moving depends on the individual's perception of the beauty. Hand drawn art often has the aesthetic attributes, and the aesthetic value of its art lies in its personalized and humanized design.

Individuality refers that an object is different from other characteristics of things. With the completion of the industrial revolution, the world entered the era of intelligent, and the contradiction between industrial production and market demand was slowly disappearing, the need of human nature was also slowly liberated. All walks of life change from the standardization of the beginning to the individual, and they need to create a unique personality image. Therefore, personalized design has broad prospects, and new media advertising design begins to focus on personalized design. In order to meet the requirements of the public to the individual, Hand drawn art with a unique visual language uses new media advertising, which has a very important value and practical significance on the design of the new media advertising

The beauty of personality is that in people's pursuit of personalized future, the internet's visual symbols will be personalized. New media advertising will be personalized and customized. The hand drawn art in the new media advertising often expresses in perceptual form, but there are other standards in the freedom, which is a combination of the theme of the graphics, and its unique personalized visual language often more clearly expresses the beauty while design of computer software can't reach.

The perspective of business marketing. Social development needs to rely on the market economy, and in today's society, the design of advertising is mainly to promote the business of products in the market economy situation, so, the design of the advertising is essentially for the market economy. In the modern society, the brand effect is filled with all over the world, for a company, its brand influence directly determines the future development of the enterprise. Good brand can enhance the competitiveness of enterprises and bring more economic returns to the enterprise, so the simple and personality of the hand drawn art has a very important role on the promotion of the brand. Hand drawn graphics not only need to have the artistic aesthetic, but also closely combined with the actual performance of the product. Hand drawn art not only inherits the traditional culture, but also needs to improve their business value. Therefore, in the promotion of brand, it needs to combine hand drawn art with high quality of new media advertising, improving corporate brand influence to gain more business value for the enterprise.

\section{Summary}

Along with the development of the digital technology, the form and the creation way of the hand drawn art has gradually changed. The development of Internet technology and the pursuit of personalized advertising has changed greatly on the simple image of the hand drawn graphics mode. The visual advertisements in Internet and new media mainly rely on the image transmission information, so people in the new era of reading are constantly changing on the image aesthetic art, and new media advertising maintains the original power, in order to improve its core competitiveness. This paper studies the form and visual features of the hand drawn art, to further explore the innovative application value in the new media advertising design, the research shows that the development of the hand drawn art has a very important practical significance on the development of new media advertising design.

\section{References}

[1] W.T. Dong. Study on the value of hand drawn graphic art in the design of web advertising. Zhejiang Gongshang University, 2014: 1-12. 
[2] L. Lan. Study on the innovation and application of hand drawn illustration art in the new media age. Hunan Normal University, 2013: 3-11.

[3] S.Y. Man. The value discussion of hand-painted graphic art in network advertisement design. Science Journal, 2015(9):154-155.

[4] R.Y. Li. The analysis of the interactive network advertising from the perspective of the audience. Young reporters, 2014(20): 7-79.

[5] J. Wang. The design and implementation of web advertising management system based on J2EE. Lanzhou University, 2014: 1-12.

[6] H.J. Lv, M. Cheng. Research on the network advertisement effect evaluation based on the perspective of consumer psychology. Journal of Nanjing agricultural university, 2015(4): 46-50.

[7] Q.L. Zhang. Research on the new media age internet advertising. Huazhong Normal University, 2014(2): 67-68.

[8] T. Ma, S. Zhang. The fusion development trend of audio-visual new media and traditional media. Wireless internet technology, 2014(6):132-133.

[9] M.X. Tian. Research on the personalized art expression strategy of hand drawn animation in the new media age. Art and design, 2014(8): 80-82.

[10] S.H. Weng. Research on the performance of hand drawn animation art in the new media age. Art \& technology, 2014(6): 415-416.

[11] L.F. Xu. The application value of exploring the hand drawn graphic arts in graphic design. Xi'an Academy of Fine Arts, 2014: 1-8.

[12] S.G. Lu. The application value of hand graphic arts in graphic design. Curriculum education research, 2015(9): 224-225.

[13] H.J. Gao. A comparative study on the development trend of new media and traditional media. Electronic production, 2014: 17-24.

[14] X.H. Zhang. The online advertising psychological characteristics and online advertising creation. Jinan University, 2015: 2-6.

[15] X. Yang. The application research on the illustration hand drawing value and its packaging design. Nanchang University, 2013: 5-11. 